\title{
Automation and prothrombin time: a United Kingdom field study of two widely used coagulometers
}

\author{
J M Thomson, D A Taberner, L Poller
}

\begin{abstract}
Current performance in the prothrombin time (PT) of the two main United Kingdom coagulometer/thromboplastin systems was assessed in a field survey. Twenty abnormal samples covering a wide spectrum of International Normalised Ratio (INR) were distributed to users of the KC4/KC10 and Coag-a-Mate instruments. Coagulometer results were compared with those of the manual method. A substantial minority with each system showed good agreement with the manual reference values. There was, however, a considerable variation between instruments, meaningful in clinical terms, evidenced by varying regression slopes and local system International Sensitivity Indices (ISI). For intense anticoagulation ( 3.0 to 4.5 INR) a larger dose of warfarin is needed with the Coag-a-Mate than with the KC instruments. With a manual INR of 4.0 the KC instruments tended to give longer $P T$ (mean INR + 0.3); the Coag-a-Mate PT was generally shorter (mean INR -0.1). With both systems the mean normal PT were shorter than the manual but the degree of shortening did not parallel that of the abnormal samples. This effect undermines the use of a simple prothrombin ratio and of an INR value derived from it, based on a manual ISI. The use of a system related ISI cannot, however, be recommended until local instrument variables are controlled.
\end{abstract}

About $50 \%$ of United Kingdom hospital laboratories use a coagulometer for prothrombin time (PT) testing and about half of these use one of two types of instrument; KC4/ $\mathrm{KCl0}$ (Amelung) and Coag-a-Mate $\times 2 / \times \mathrm{C}$ (Organon Teknika). We have previously shown in National External Quality Assessment Surveys (NEQAS) that coagulometers have an effect on the International Normalised Ratio (INR) which varies not only among the different types of coagulometer but also within the instruments. ${ }^{1}$ These data were obtained retrospectively from NEQAS comprising two or three test plasmas per survey, conducted over two years. The present prospective study was designed to obtain contem- porary comprehensive data on the instrument effect on PT and INR values.

\section{Methods}

All centres using either the $\mathrm{KC} 4 / \mathrm{KCl}$ or the Coag-a-Mate $\times 2 / \times \mathrm{C}$ with the Manchester Reagent (MR) were invited to take part. The study was confined to one thromboplastin because other reagents are not used in sufficient numbers in the United Kingdom for statistical analysis with these instruments and because our previous study had shown that there was a thromboplastin/instrument interaction. ${ }^{1}$

A range of 20 lyophilised test plasmas was provided for all participants together with a supply of MR. Samples were taken from 10 stabilised patients, who had been treated with coumarin, with a wide scatter of International Normalised Ratios (INR) values, and from 10 artificially depleted samples giving a similar spectrum of INR. Because of the limited volumes of some of the patient donations, two separate series of 20 abnormal test plasmas were provided for the two test systems, but each gave a similar span of INR values and an identical mean manual PT of 47 seconds. Nine of the plasmas were common to each set of 20. Participants were asked to test these samples using their customary KC or Coag-aMate technique. The stated mean normal prothrombin time (MNPT) value at each centre was requested. The $\mathrm{KC}$ study was divided into two parts consisting of 10 adsorbed plasmas on the first occasion and 10 coumarin samples one month later. The purpose was to determine the consistency of performance over time and whether the type of deficient plasma influenced the result. For the Coag-a-Mate users all 20 plasmas were issued with MR on one subsequent occasion.

The results obtained by participants with each test plasma were compared with those obtained by the manual method. The latter was determined from sufficient replicate testings by six laboratory staff at the United Kingdom Reference Laboratory to ensure a mean coefficient of variation between $1.25 \%$ at the lower end of the INR scale and a maximum of $3.5 \%$ at higher values as the coefficient of variation of the PT depends on its degree of prolongation. ${ }^{23}$

The results of the PT of the 20 plasmas with each instrument were plotted against the manual results to provide linear and logarithmic orthogonal regression slopes. The PT were transformed into INR in two different
Correspondence to: L Poller

Accepted for publication 8 February 1990 
ways: (a) based on the locally derived prothrombin ratio using the stated local MNPT and the International Sensitivity Index (ISI) of $\mathrm{MR}$; and (b) based on the prothrombin ratio derived using the manual MNPT and the MR ISI value. The mean manual MNPT was determined using the geometric mean of 20 normal samples and the ISI of the two batches of MR were checked independently in a full calibration exercise against the second primary WHO International Reference Preparation, human plain BCT/253. ${ }^{4}$

For each centre the percentage deviations between instrument and manual INR were calculated for each test plasma using the local instrument MNPT or the mean manual normal value provided with $M R$.

An approximate ISI for each local instrument/system was determined from the logarithmic orthogonal regression slopes of the PT results of the 20 test plasmas and the ISI of MR.

\section{Results}

The manual MNPT with the two batches of MR was 15.9 seconds. This was identical with the value provided by the manufacturer. The stated ISI values of these batches were confirmed within the recommended 3\% limit of the WHO calibration procedure.

All forty four centres using the $\mathrm{KC}$ returned results and 25 of 27 of the Coag-a-Mate system users. Figures 1a-1d show the arithmetic orthogonal regression slopes with the two systems. The mean values expressed as PT (seconds) for the two instruments, compared with the manual results, are given in fig la. Figures $1 \mathrm{~b}$ and $1 \mathrm{c}$ show the slopes for $\mathrm{KC}$ users on adsorbed and coumarin plasmas tested in consecutive months. These were consistent between dates and source of plasma in $60 \%$ of the centres. Both systems showed a considerable scatter of slopes (figs $1 \mathrm{~b}-1 \mathrm{~d}$ ). The $\mathrm{KC}$ gave good overall agreement with the manual method only between 25-30 seconds whereas with the Coag-a-Mate there was no point of coincidence between manual and instrument results. With the $\mathrm{KC}$, the PT with the abnormal plasmas tended to be up to 25 seconds shorter than the manual but longer with higher values. In contrast, the Coag-a-Mate PT were consistently shorter than the manual method over the range of test plasmas, although the
Figure 1 (A) Mean arithmetic orthogonal regression lines of the $K C$ (----), Coag-a-Mate (.........), and the line of coincidence ( between manual and instrument results. (B) Individual centre's arithmetic orthogonal regression lines for $K C$ with adsorbed plasmas. (C) Individual centre's arithmetic orthogonal regression lines for $K C$ with coumarin plasmas. (D) Individual centre's arithmetic orthogonal regression lines for Coaga-Mate (adsorbed and coumarin plasmas combined).
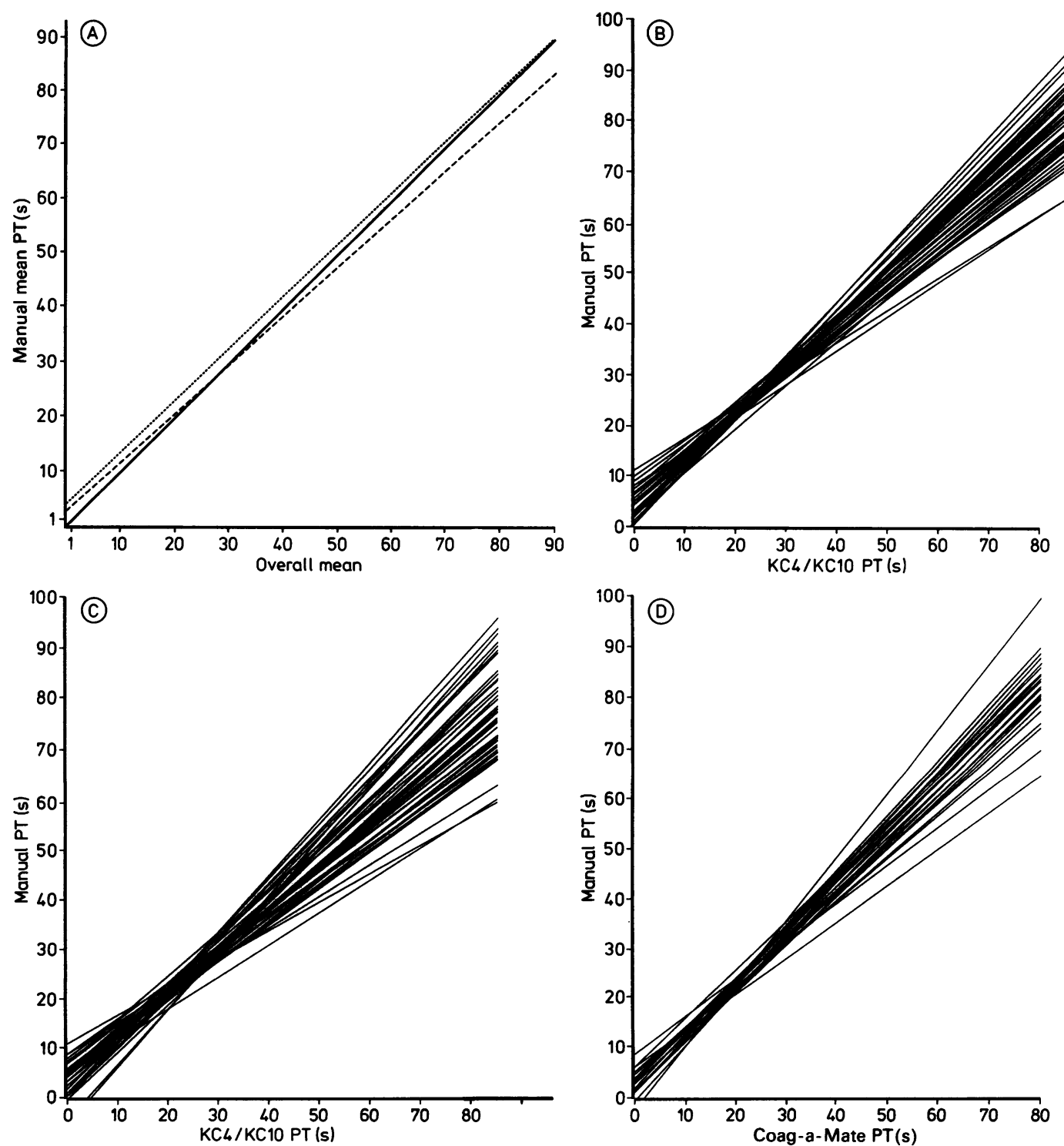
Figure 2 Distribution of stated coagulometer MNPT.
Figure 3 Relation between stated MNPT and mean abnormal $P T$ for each Coag-a-Mate.
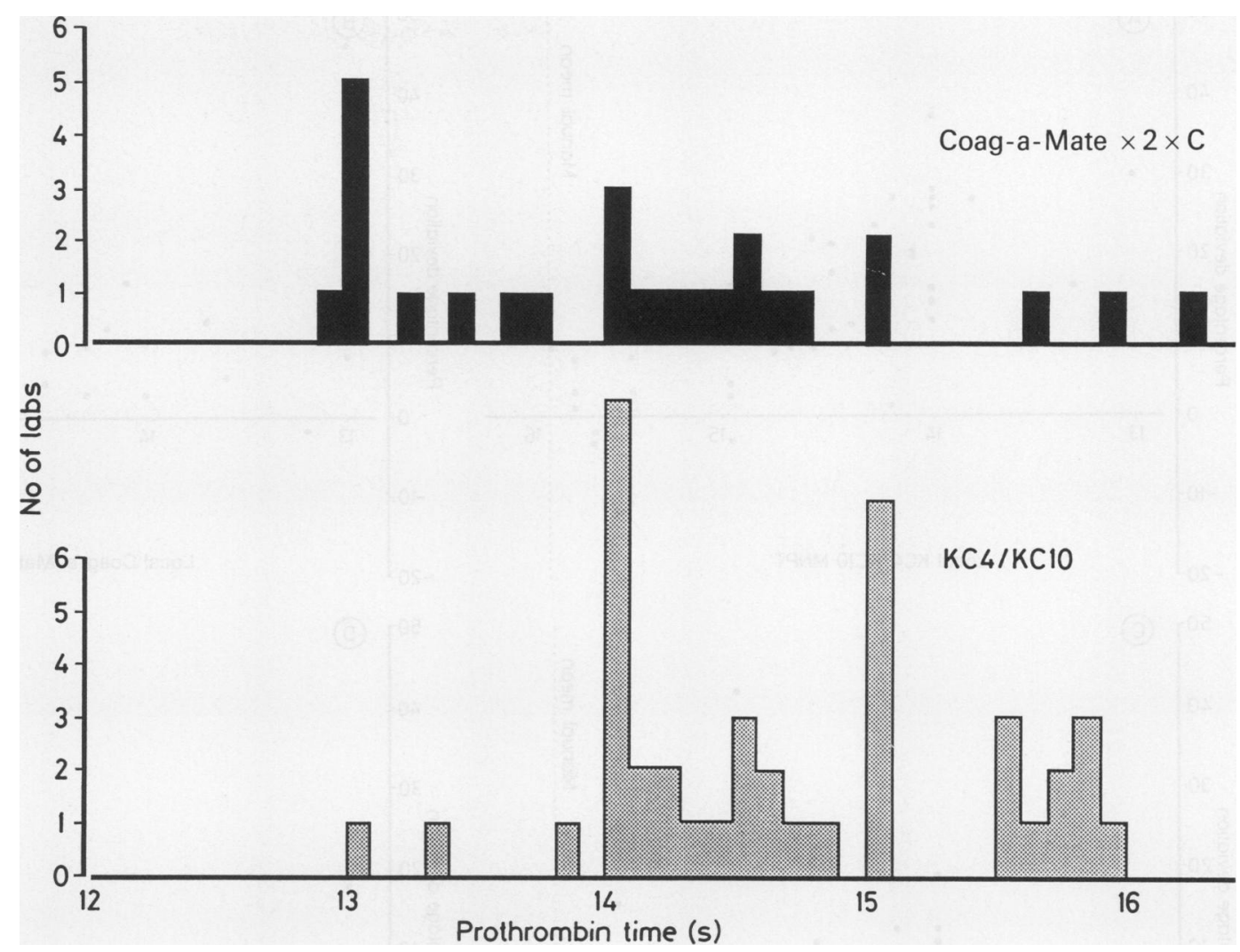

percentage deviation diminished with increasing PT.

Figure 2 shows that the stated MNPT with both systems were shorter than that of the manual method but with considerable variation between instruments. With the $\mathrm{KC}$, the shortening was also observed with mild defects, but there was prolongation with intermediate and more severe coagulation defects-that is, a

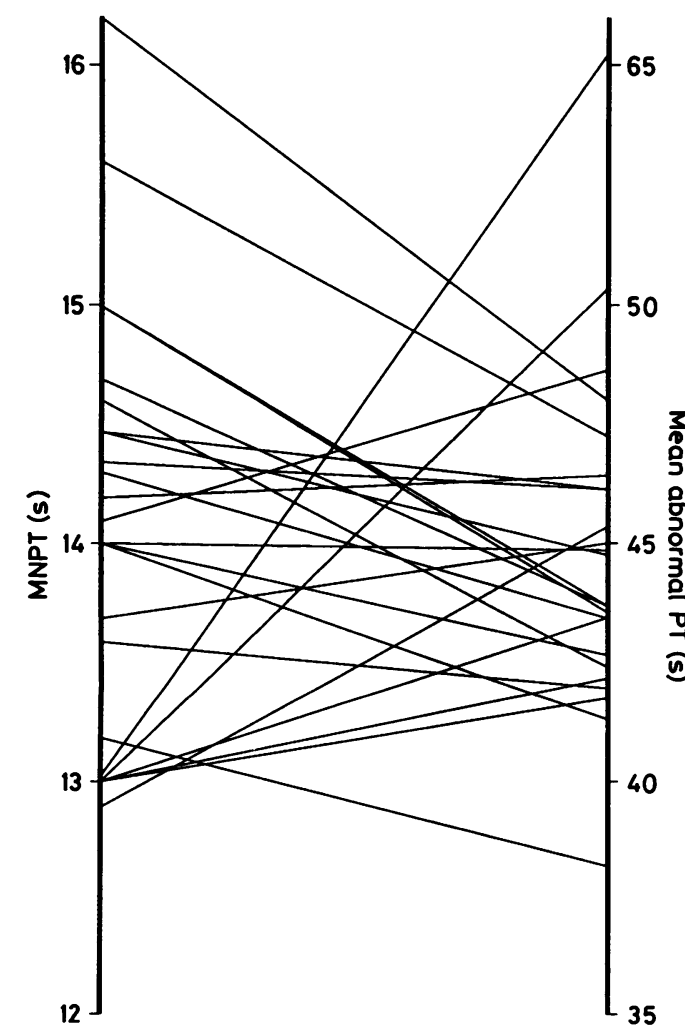

"crossover" effect. This is shown in the table which provides an assessment of the deviation in seconds from the manual at three representative levels of anticoagulation, approximating to INR of $2 \cdot 1,3 \cdot 2$, and $4 \cdot 5$. The percentage showing a shortened PT with the $\mathrm{KC}$ fell abruptly as the coagulation defect increased. In contrast, with the Coag-a-Mate the PT was about two seconds shorter than that of the manual across the range of test plasmas. The MNPT reported with most Coag-a-Mate instruments were $<15.9$ seconds, but the degree of acceleration did not parallel the observed shortening of the abnormal test samples (fig 3).

The instrument effect on INR values, calculated from the local MNPT or the manual mean provided by the manufacturer, were determined. With the KC system, the mean percentage deviation obtained with the local instrument MNPT was +15.02 but was reduced to +4.76 when the manual mean value provided by the manufacturer was used ( $p<$ 0.001). With the Coag-a-Mate, the difference

Differences from manual times (seconds) obtained from individual regression lines

\begin{tabular}{|c|c|c|c|c|c|}
\hline \multirow[b]{2}{*}{$\begin{array}{l}\text { Mean } \\
\text { deviation from } \\
\text { manual PT }\end{array}$} & \multirow{2}{*}{$\begin{array}{l}\begin{array}{l}\text { Manual } \\
P T \\
\text { (seconds) }\end{array} \\
30 \\
45 \\
60\end{array}$} & \multicolumn{2}{|c|}{$\begin{array}{l}\text { KC4/KC10 } \\
(\text { seconds) } \\
(\%)\end{array}$} & \multicolumn{2}{|c|}{$\begin{array}{l}\text { Coag-a-Mate } \\
\times 21 \times C \\
(\text { seconds })(\%)\end{array}$} \\
\hline & & $\begin{array}{l}-0.2 \\
+1.6 \\
+4.0\end{array}$ & $\begin{array}{l}-0.7 \\
+3.6 \\
+6.7\end{array}$ & $\begin{array}{l}-2.5 \\
-2.4 \\
-1.8\end{array}$ & $\begin{array}{l}-8 \cdot 3 \\
-5 \cdot 3 \\
-3 \cdot 0\end{array}$ \\
\hline $\begin{array}{l}\text { Range of } \\
\text { difference from } \\
\text { manual PT }\end{array}$ & $\begin{array}{l}30 \\
45 \\
60\end{array}$ & \multicolumn{2}{|c|}{$\begin{array}{c}-3 \cdot 3 \text { to }+3 \cdot 7 \\
-4.9 \text { to }+10.9 \\
-6.4 \text { to }+20.0 \\
\%\end{array}$} & \multicolumn{2}{|c|}{$\begin{array}{l}-6 \cdot 1 \text { to }+2.9 \\
-7.9 \text { to }+7.4 \\
-11 \cdot 1 \text { to }+12.9 \\
\%\end{array}$} \\
\hline $\begin{array}{l}\text { ercentage } \\
\text { lorter than } \\
\text { lanual PT }\end{array}$ & $\begin{array}{l}30 \\
45 \\
60\end{array}$ & & $\begin{array}{l}53 \cdot 5 \\
30 \cdot 2 \\
23 \cdot 3\end{array}$ & & $\begin{array}{l}96 \cdot 2 \\
84 \cdot 6 \\
73 \cdot 1\end{array}$ \\
\hline
\end{tabular}



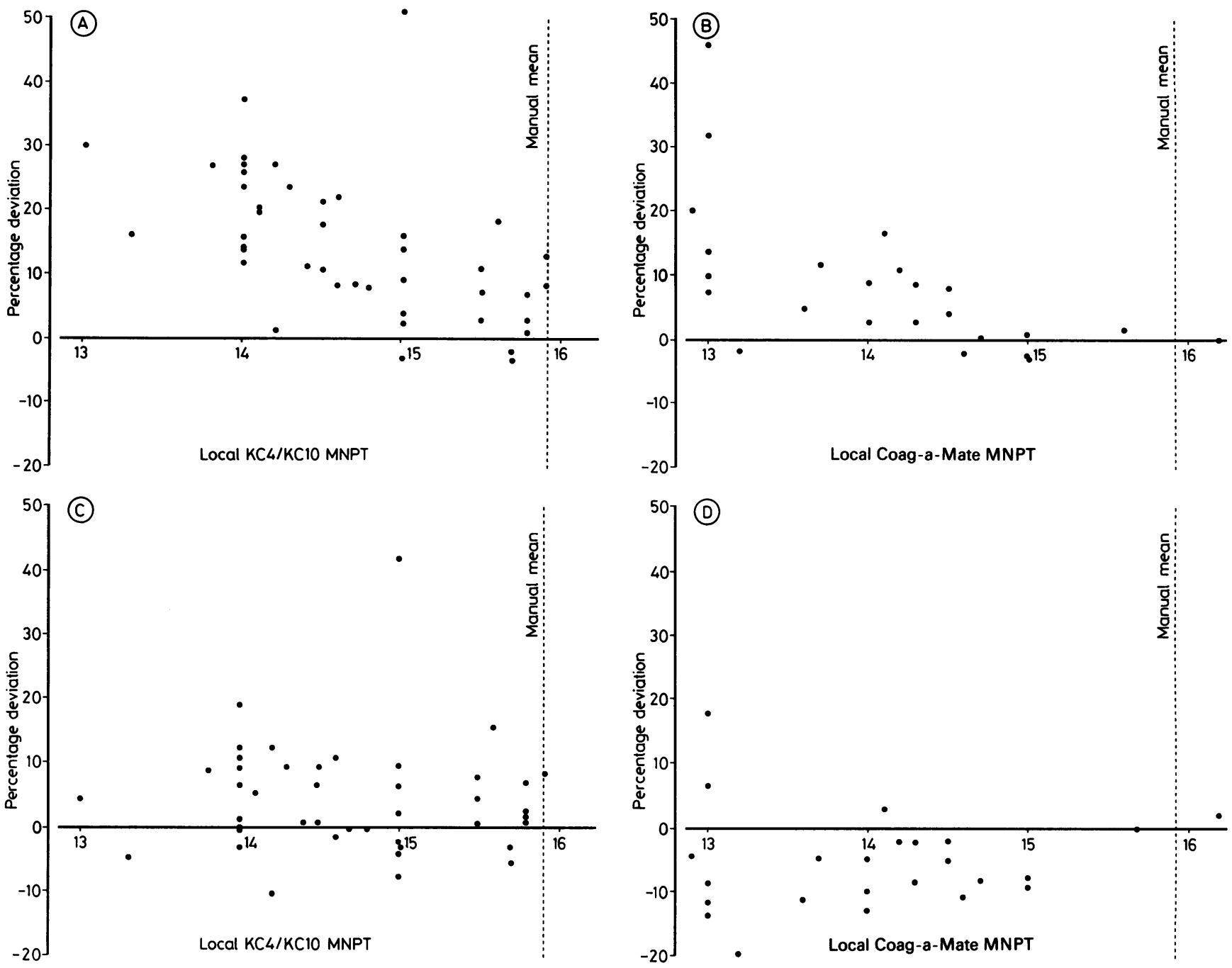

Figure 4A-D Mean percentage deviation of coagulometer INR from manual for each centre plotted against stated local MNPT.

(A) When local MNPT is used to derive INR for $K C$

(B) When local MNPT is used to derive INR for Coag-a-Mate.

(C) When manufacturer's stated manual MNPT is used to derive INR for KC.

(D) When manufacturer's stated manual MNPT is used to derive INR for Coag-a-Mate.

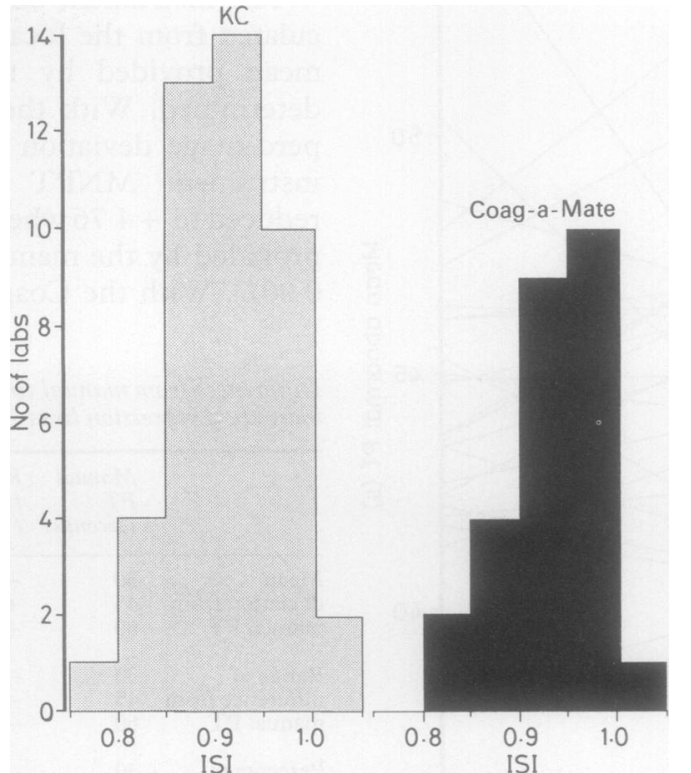

Figure 5 Effect of instruments on ISI values, discounting thromboplastin ISI. between the two procedures was smaller and not significant. The local MNPT method to derive the INR overestimated the value by $8.3 \%$ whereas using the stated MNPT with MR, the INR were underestimated by $5 \cdot 2 \%$.

When the instrument INR is derived from the prothrombin ratio using the local stated MNPT, with both the KC and Coag-a-Mate, the shorter the reported local MNPT the greater the percentage deviation of the instrument INR from the manual INR (figs 4a and $b$ ). This negative correlation was significant at the $1 \%$ level for the two systems. In contrast, there was no relation between the deviation and the MNPT when the instrument INR was derived from the manufacturer's stated manual MNPT (figs 4c and d).

A wide range of approximate ISI values for each centre's automated system, derived from the logarithmic orthogonal regression analysis, is shown in fig 5, reflecting the instrument differences and ignoring the thromboplastin effect. 


\section{Discussion}

The multicentre field survey described in this report has evaluated the current performance of the two most widely used coagulometer systems in the United Kingdom and has shown considerable variability within instruments with both systems as well as deviations from the manual result. In the British system for oral anticoagulant control recommended therapeutic ranges have been derived from clinical trials and cumulative experience with the manual PT technique. For this reason the manual technique has been the reference PT method against which automated procedures have been assessed-the longstanding United Kingdom practice.

Previous coagulometer studies have been based on assessments of single instruments of one or more types at one centre ${ }^{6}$ or with groups of instruments by retrospective analysis of cumulative data obtained over an extended period in national external quality assessment. This type of datum is therefore limited and does not reliably assess performance in clinical practice. Assessments of single instruments provide no indication of the variation between instruments with a multiplicity of coagulometers of the same type. Retrospective external quality assessment based on limited data on each occasion may not reflect current performance and is inevitably affected by instrument changes and adjustments which occur over an extended period.

Our study shows that a substantial minority of the $\mathrm{KC} / \mathrm{Coag}$-a-Mate users obtained good agreement with the manual reference values independently established over the range of abnormal test plasmas. This indicates that close approximation to the manual result is possible using the two systems in patients given coumarin. The overall trend with the coagulometers, however, is to accelerate the normal PT, although the extent varies considerably among centres. The stated MNPT values for the two systems ranged between 13.0 and 16.2 seconds with a geometric mean of 14.6 seconds for KC and 14.0 for the Coag-a-Mate, compared with 15.9 which is the manual geometric mean for MR. Unexpectedly, the acceleration observed with the depleted samples did not parallel the shortening of normal values assessed from the stated locally derived MNPT. With the KC at levels less than therapeutic the PT was generally shorter than that of the manual, but for values over 30 seconds the overall tendency was for results to become progressively longer by comparison. A different pattern was observed with the Coaga-Mate. The trend to shorter PT became relatively less pronounced with higher values. The mean percentage deviation for INR calculated from the local instrument MNPT for the $\mathrm{KC} / \mathrm{KC} 10$ was $+15 \cdot 02$, and that for the Coag-a-Mate $+8 \cdot 34$. The mean percentage deviation calculated from the manufacturer's stated manual MNPT was +4.76 for the KC4/ $\mathrm{KC10}$ and $-5 \cdot 23$ for the Coag-a-Mate. These discrepancies, unless corrected, combined with the degree of variation of locally reported
MNPT to undermine confidence in a ratio based control system.

A further important observation related to the derivation of the INR is that the shorter the stated local MNPT the greater the disagreement between instrument and manual INR. The negative correlation of the local MNPT and the percentage deviation from the manual INR (figs $4 a$ and $b$ ) clearly shows that the derivation of INR values using the locally derived normal value with either test system does not correct for the instrument effect. Ideally, therefore, the instrument should be adjusted to give a local MNPT as close as possible to that of the manual. The variation in the MNPT is most likely to depend on the instrument. Irrespective of the cause, the use of the locally derived instrument MNPT is shown in this study to introduce considerable error in derivation of INR values.

The above effects on the normal values and the differences in orthogonal regression slopes of the abnormal samples given in figs 1 and 2 might indicate the need for adjustment of the manually derived ISI to take into account the instrument effects. The extent of variability between instruments illustrated by the differences in slopes and MNPT show that it is currently impossible to assign a system specific ISI applicable to all instruments of the same type. The great degree of variation between the centres can be gauged by the instrument specific local ISI values determined from logarithmic orthogonal regression slopes (fig 5).

In clinical terms the mean deviation from the manual INR value with both instruments is not great, the respective figures being -0.1 with the Coag-a-Mate and +0.3 with the $\mathrm{KC}$ for a manual INR of 4.0. Slightly more oral anticoagulant would be prescribed with Coaga-Mate control and less with $\mathrm{KC}$ control. With some Coag-a-Mate and $\mathrm{KC}$ instruments, however, effects on dosage may be much more noticeable than these mean figures suggest.

The derivation of an ISI value based on a local full calibration of a single instrument system might correct these dosage problems. The limitations of this approach are the excessive demands on normal and patient samples for a full calibration and the inherent error of a single calibration at one centre. Furthermore, a calibration exercise necessarily entails parallel observations using the manual reference technique. This may not be feasible in all centres.

The two part KC study addressed two additional issues-whether performance of the coagulometers was consistent over a period of time and whether artificially adsorbed abnormal plasmas gave the same order of result as samples from patients given coumarin. The overall findings confirmed both these. Thus the availability of lyophilised abnormal plasmas calibrated by the manual method in PT and INR, together with lyophilised normal samples, may provide a mechanism for adjustment of instruments to give results comparable with those of the manual technique. This should improve the unacceptable variability 
currently found within instruments in this study with the two major coagulometer systems used in the United Kingdom and might then allow system based ISI to be developed.

1 Poller L, Thomson JM, Taberner DA. Effect of automation on prothrombin time test in NEQAS survey. J Clin Pathol 1989;42:97-100.

2 Poller L, Taberner DA, Thomson JM, Darby KV. A survey of the prothrombin time results in NEQAS exercises 1980-1987. J Clin Pathol 1988;41:361-4.
3 Taberner DA, Poller L, Thomson JM, Darby KV. Effect of international sensitivity index (ISI) of thromboplastins on precision of international normalised ratios (INR). J Clin Pathol 1989;42:92-6.

4 World Health Organisation Expert Committee on Biological Standardisation: 34th Report. WHO Technical Report Standardisation: 34th Report. WHO Technical Report

5 World Health Organisation Expert Committee on Biological Standardisation: 33rd Report. WHO Technical Report Series. Geneva: WHO, 1983:1-105.

6 D'Angelo A, Seveso MP, D'Angelo SV, et al. Comparison of two automated coagulometers and the manual tilt tube method for the determination of prothrombin time. Am J Clin Pathol 1989;92:321-8. 